
sophisticated public information to avoid

SUBJECT AREAS:

EVOLUTION

ANIMAL BEHAVIOUR

REPRODUCTIVE BIOLOGY

NEUROSCIENCE

Received

20 June 2012

Accepted

19 September 2012

Published

25 October 2012

Correspondence and requests for materials should be addressed to A.L. (al@adeline-loyau. \section{mated males}

\author{
Adeline Loyau ${ }^{1,2,3}$, Simon Blanchet ${ }^{1,2,3}$, Pauline Van Laere' , Jean Clobert ${ }^{1} \&$ Etienne Danchin ${ }^{2,3}$
}

${ }^{1}$ CNRS; Station d'Ecologie Expérimentale du CNRS à Moulis, USR 2936, 2-4 Route du CNRS; 09200 Saint Girons, France, ${ }^{2}$ CNRS, Université Paul Sabatier, ENFA; Laboratoire d'Evolution et Diversité Biologique (EDB), UMR 5174, 118 Route de Narbonne, Bât. 4R3, 31062 Toulouse Cedex 9, France, ${ }^{3}$ Université de Toulouse, UMR 5174, 118 Route de Narbonne, Bât. 4R3, 31062 Toulouse Cedex 9, France.

Semen limitation (lack of semen to fertilize all of a female's eggs) imposes high fitness costs to female partners. Females should therefore avoid mating with semen-limited males. This can be achieved by using public information extracted from watching individual males' previous copulating activities. This adaptive preference should be flexible given that semen limitation is temporary. We first demonstrate that the number of offspring produced by males Drosophila melanogaster gradually decreases over successive copulations. We then show that females avoid mating with males they just watched copulating and that visual public cues are sufficient to elicit this response. Finally, after males were given the time to replenish their sperm reserves, females did not avoid the males they previously saw copulating anymore. These results suggest that female fruit flies may have evolved sophisticated behavioural processes of resistance to semen-limited males, and demonstrate unsuspected adaptive context-dependent mate choice in an invertebrate.

ncreasing attention is being paid to plasticity in mate choice because it can profoundly affect the strength and direction of selection ${ }^{1-4}$. Plasticity in decisions such as mate choice is crucial to permanently track and adaptively respond to fluctuations in environmental conditions, including variations in the social context. This is particularly the case in species where males may be semen-limited. In these species, natural selection should favour females that can detect potentially semen-limited males. Females, for instance, may watch individual male's recent mating histories and use such social information to avoid recently mated males. Accordingly, theoretical considerations suggest that the use of social information in mate choice depends on the specific conditions and associated pay-offs ${ }^{5}$. However, to which extent females can adaptively modify their mate choice according to social information remains an open issue. So far, the use of social information for mate choice has been largely studied in the context of mate choice copying ${ }^{6}$, in which a female's probability of choosing a given male increases if another female has previously chosen that male ${ }^{7,8}$, implying that mate choice copying may not always be adaptive in species with high rates of semen-limited males.

Semen limitation is thought to be common in sexual species in which male can mate with several females successively ${ }^{9-10}$. Semen is a complex fluid composed of seminal liquids containing sperm cells as well as additional products including proteins, lipids, carbohydrates, minerals, vitamins. Therefore, semen limitation can result from shortage in various semen components, predominantly sperm cells ${ }^{9-10}$. In species where successive mating are common, females may not receive enough sperm to fertilize all of their eggs, thus incurring costs in terms of reduced number of offspring ${ }^{9-10}$. In the fruit fly, Drosophila melanogaster, successive mating lead to the depletion of male accessory glands ${ }^{11-16}$, which produce accessory proteins (Acps) influencing both male and female reproductive capacities, such as sperm transfer, sperm storage, female receptivity, female ovulation and early egg fertility ${ }^{14}$. Female fruit flies mated to semen-limited males therefore produce lowered numbers of offspring ${ }^{11-16}$, as we also found here (Fig. 1). Moreover, mating is assumed to impose costs as the time spent searching and assessing mate quality increases predation risks ${ }^{17-18}$, and as copulation reduces immunity ${ }^{19}$. Costs of mating with semen-limited males are exacerbated when copulation is extensively long compared to individual lifespan, and when males and females have highly divergent interests in reproduction as in the fruit fly ${ }^{20-22}$ (where mating reduces female ability to re-mate by inducing a refractory period, and reducing female lifespan ${ }^{20,23}$ ). Hence, the resulting additional costs of mating are not compensated for by offspring production when mating with semen-limited males. 


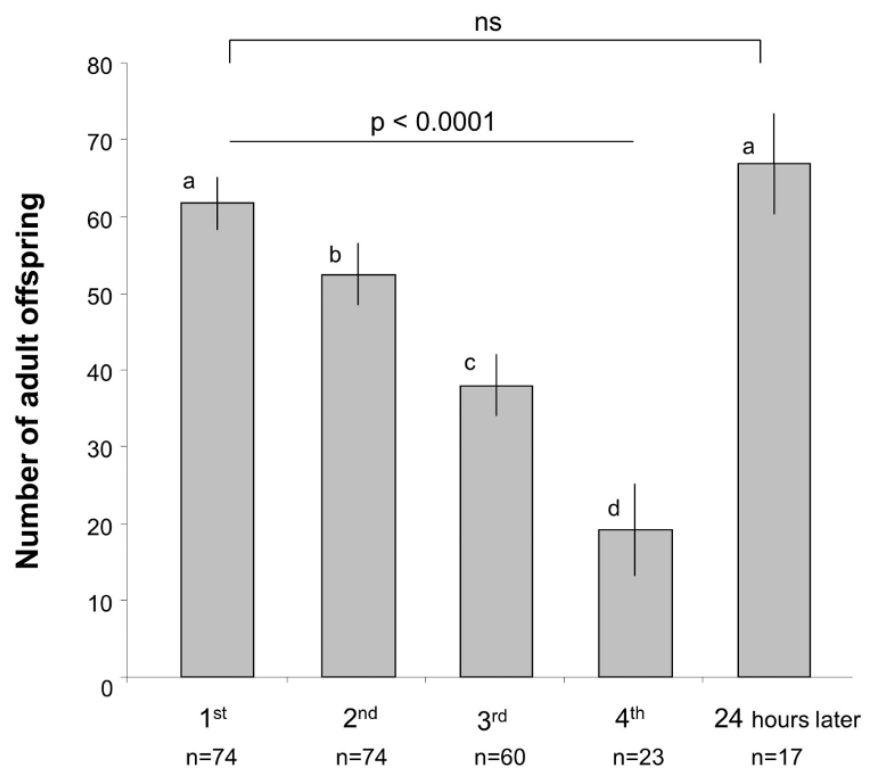

Copulation number by a given male

Figure $1 \mid$ Differences in adult offspring production over 5 successive copulations by males. $\mathrm{Y}$ axis: number of offspring imagos produced by the $\mathrm{n}^{\text {th }}$ copulation. 24 hours refer to the $5^{\text {th }}$ copulation that occurred 24 hours after the $4^{\text {th }}$ copulations. Number of offspring imagos significantly dropped along the first four copulations (GLM: $\mathrm{F}_{1,172}=44.49 ; \mathrm{p}<0.0001$ ) but returned to the initial level after 24 hours (GLM: $\mathrm{F}_{1,90}=0.43$, $\mathrm{p}=0.5122$ ). Different letters indicate significant differences (one-tailed post-hoc tests).

Evolutionary theories predict that females should evolve resistance against semen-limited males ${ }^{22,24}$. Females should therefore use any available piece of information to avoid semen-limited males. To date, only a few empirical studies have analyzed the influence of semenlimitation on female mate choice ${ }^{25-27}$. Female cockroaches Nauphoeta cinera and female stone crabs Hapalogaster dentata, for instance, avoid semen-limited males when information about male's semen availability is accessible through chemical cues $^{25-26}$. Interestingly, in Drosophila bifurca, a fruit fly with giant sperm in which both sexes produce only few gametes, females do not appear able to discriminate between semen-limited and virgin males based on chemical $\mathrm{cues}^{27}$.

The information extracted from the observation of sexual interactions is public ${ }^{28-29}$. Watching potential mate's copulations has been shown to influence mate choice in the form of mate choice copying in various vertebrate and invertebrate species ${ }^{28-32}$, including Drosophila melanogaster ${ }^{8}$. More specifically, when D. melanogaster females are given the choice between two virgin, not semen-limited, males they copulate preferentially with males of the phenotype that they previously saw copulating over males of another phenotype that they saw being rejected ${ }^{8}$. Here, we hypothesize that females also use public information extracted from previous copulations to avoid mating with semen-limited males, and that this preference is context-dependent given that semen-limitation is temporary.

We use a series of four experiments to test these hypotheses in the fruit fly D. melanogaster. In this species, the probability to encounter semen-limited males is high because males have big sperm, are semenlimited after successive matings ${ }^{11-16}$ and males keep courting and mating even after four or five successive copulations ${ }^{12,33}$. We first show that the number of offspring gradually decreases over successive copulations (Fig. 1). Then, we test whether fruit fly females use public information on potential mates' copulation history by avoiding mating with a male that they watched copulating once.
We used a row of three transparent plastic vials (Fig. 2) separated by microscope cover glasses. A virgin observer female was placed in the central one. During the demonstration phase, she could see social interactions occurring in the two peripheral vials. We manipulated the nature of the public information provided to the observer female by varying the kind of social interaction occurring in the two peripheral vials. In all situations, males differed by the colour (green or pink) of the powder that dusted their thorax and back, which we used to individually recognize them. During the subsequent copulation test, attractor females and the glass partitions were removed and we recorded the male with which the observer female copulated.

\section{Results}

In our first experiment, one male was placed with a virgin female, the other with a refractory female who just copulated. The former copulated rapidly (Mated males) while the other only unsuccessfully courted the attractor female (Unmated males). The observer female thus witnessed a male that copulated and another that did not copulate during the demonstration phase. After such a demonstration, observer females that watched the interactions mated preferentially with the Unmated males (Fig. 3, left bars). Similarly, males that did not encounter females (Virgin-control) were preferred over Mated males when observer females watched the demonstration (Fig. 3, middle bars). Finally, observer females did not discriminate between Virgin-control and Unmated males (Fig. 3, right bars). This first experiment suggests that females have the capability to detect males that just copulated to avoid copulating with them. This could be because courting and mating can have exhausted the males during the demonstration phase, because females can smell copulation odours on males and/or because they watched and remembered which male had just copulated.

Our second experiment was run in parallel with the first one to tell these three alternatives apart. We performed the same experiment except that the demonstration phase was conducted separately so that females could not observe Mated and Unmated males courting and mating. In this way, when choosing between males, observer females could smell body odours on males or detect differential male behaviour but had not visually witnessed which of the two males had copulated or not. In such conditions, females did not show any preference for Unmated over Mated males (Fig. 4, left bars), nor did they prefer Virgin-control over Unmated males (Fig. 4, right bars). However, there was a marginally non-significant tendency for Virgin-controls to be preferred over Mated males (Fig. 4, middle bars), suggesting that some females may have been able to detect virginity using odour cues and/or male behaviour. By comparison with Experiment 1, this second experiment strongly suggests that, in most cases, differential male behaviour or odours of sex on male bodies are not sufficient to elicit the preference for unmated males observed in the first experiment. This suggests that observer females in Experiment 1 watched copulations and used it as a source of public information to avoid mating with recently mated males. The two experiments also suggest that females paid more attention to copulations than courting.

Our third experiment aims at testing whether observer females in the two previous experiments just confused males with any male of the same phenotype (colour) or whether they were able to recognise the actual individual males they saw during the demonstration. We compare female preferences according to whether or not demonstrating Mated and Unmated males were swapped or not between experimental systems just after the demonstration phase. Under these conditions, observer females did not show any preference for the swapped Unmated males (right bars in Fig. 5) but did so for unswapped males (left bars in Fig. 5). This supports the idea that females possess the cognitive ability to discriminate not only between categories of males (green $v s$. pink) as in Méry et al. ${ }^{8}$ 's experiment, 


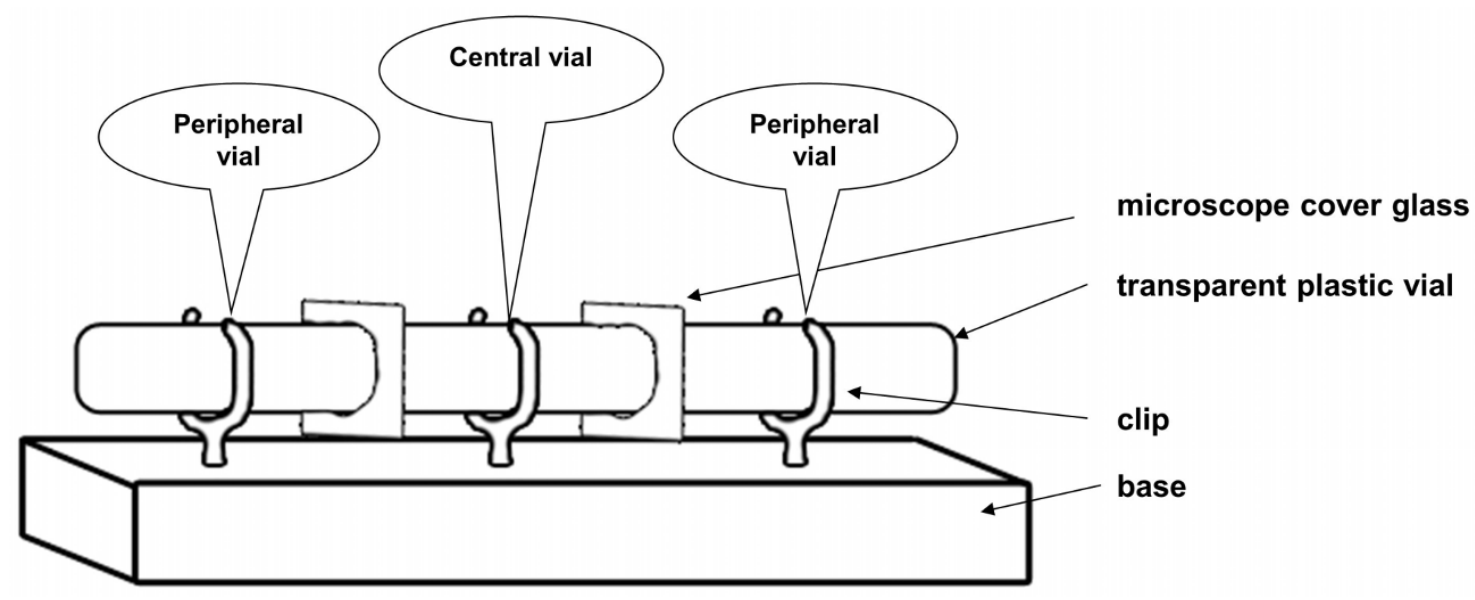

Figure 2 | Experimental set up.

but also between two given individuals (i.e. individuality recognition) as this was suggested previously ${ }^{34}$.

Our fourth experiment tackles the question of the potential benefit of that cognitive capacity. In our previous experiments, females may avoid recently mated males for several reasons. Among others, it is possible that female mating preference may be driven by the costs of semen limitation, sexually transmitted diseases (STDs) that may affect female preferences in various ways ${ }^{35-38}$, or both. To tell apart these alternatives, we exploited the fact that fruit flies have (at least) a 24 hours memory in the context of mate choice $e^{8,34,39}$. We allowed observer females to gather public information on male mating success (Mated $v s$. Unmated) and tested their choice 24 hours later. In the meantime Mated males had the time to replenish semen stocks ${ }^{12}$ (Fig. 1), but risks of STDs remained similar. Under these conditions, females did not avoid Mated males anymore (Fig. 6). Thus, female mating preferences in our experiments were probably more driven by the avoidance of semen-limitation than STDs. One could argue that this result is the consequence of memory limitation and that females simply forgot what they saw 24 hours earlier. This is one possible explanation. However, D. melanogaster has been shown to have a 24 hours memory in various contexts including mate choice $^{8,34,39}$. Furthermore, the fact that females in our experiment 4 tend to prefer Mated males over Unmated males after 24 h (Fig. 6) suggests that they retained some information and performed delayed mate choice copying. These results further show that female fruit flies are able to finely tune their mate choice decisions according to subtle differences in the context.

\section{Discussion}

Our results reveal that female fruit flies have evolved adaptive behavioural resistance to males they have seen mating, which may allow females to avoid semen-limited males. Females do so by using public information extracted from actual copulations coupled with the capacity to discriminate among two familiar males. The sophistication of these capacities recalls the complexity of mate choice in higher vertebrates $^{40-44}$. In nature $D$. melanogaster females commonly mate several times while we assessed female mating preferences and number of offspring produced from single mating opportunities. Over the female lifetime, we can expect that the potential costs and benefits of avoiding mated males, who are likely semen-limited, are additive over successive mating.

During the test phase, the female and the two demonstrator males could interact freely, therefore several potential processes could act in addition to female choice to explain pairing: male choice, male-male competition and female eavesdropping on male-male competition. However, these processes were random with respect to our experimental design and may, at best, blur our results in a way that make our interpretations conservative. Previous studies suggest that previous interaction with a demonstrator female (mating, rejection, no

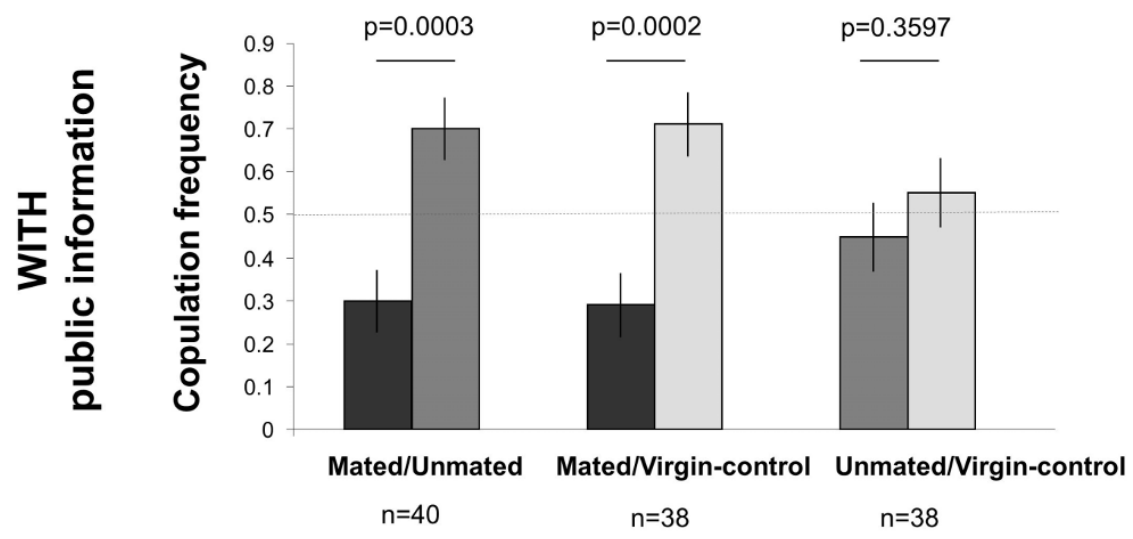

Figure 3 Drosophila melanogaster female use of social information in mate choice (Experiment 1). Females were allowed to watch male-female social interactions (Mated males copulated, Unmated males courted a female that rejected them, Virgin-control males did not encounter any female), and then were given the choice between the same two males. Observer females with social information preferred mating with the Unmated over the Mated males and the Virgin-control over the Mated males. Female showed no preference between Unmated and Virgin-control males. The horizontal dashed line corresponds to the expected values under random selection of the males. 


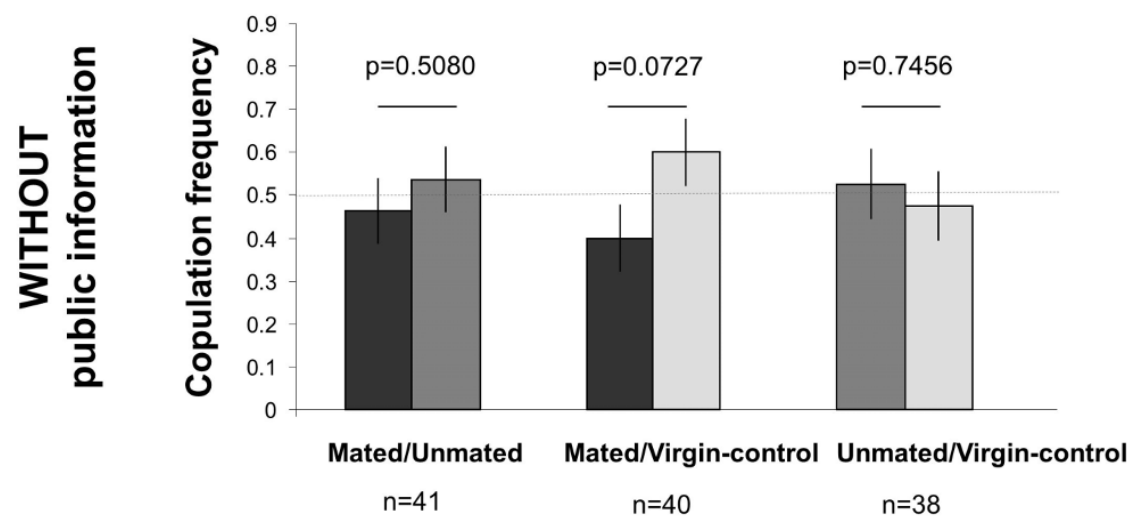

Figure $4 \mid$ Female mate choice without social information (Experiment 2, run in parallel with Experiment 1). Females were not allowed to watch male-female social interactions (Mated males copulated, Unmated males courted a female that rejected it, Virgin-control males did not encounter any female), and then were given the choice between the same two males. Observer females without visual social information showed no preference between males. The horizontal dashed line corresponds to the expected values under random selection of the males. Because experiments 1 and 2 were run in parallel, we compared results of experiments 1 and 2 for the same contrast in male type. We found a significant interaction in the case of the contrast between Mated versus Unmated males (GLM; Left panels: Mated/Unmated, male type $\times$ with vs. without information: $\left.\mathrm{Chi}^{2}{ }_{1}=4.61, \mathrm{p}=0.0318\right)$ but not for the other two contrasts in male types (Middle panels: Mated/Virgin-control, male type $\times$ with vs. without information: $\mathrm{Chi}^{2}{ }_{1}=2.11, \mathrm{p}=0.1460$; Right panel: Unmated/Virgin-control, male type $\times$ with vs. without information: $\mathrm{Chi}^{2}{ }_{1}=0.95, \mathrm{p}=0.3300$ ).

\section{SAME males}

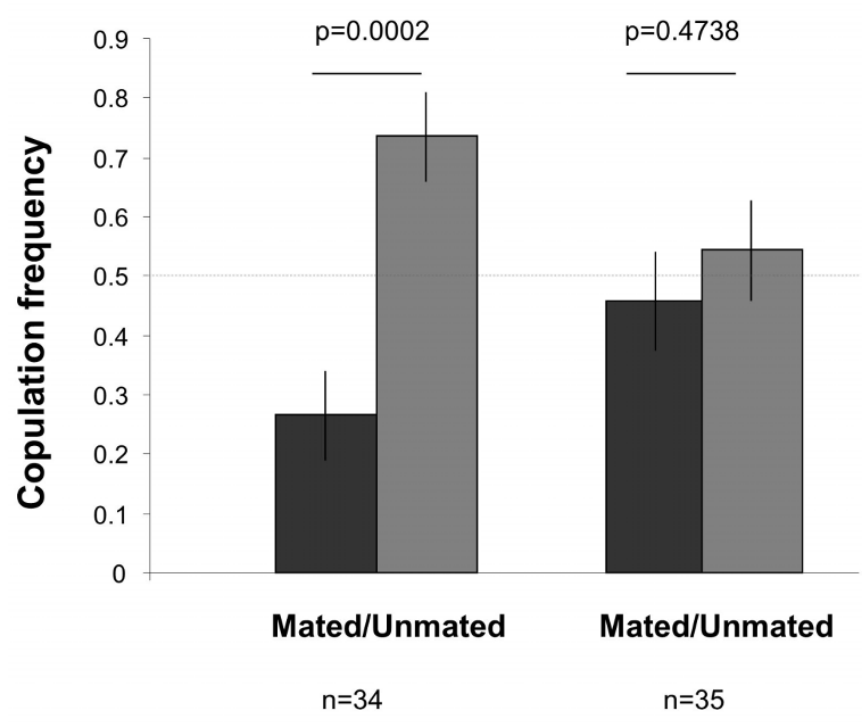

Treatment

Figure $5 \mid$ Female copulation frequency with Mated and Unmated males according to whether males were not changed between the demonstration and test phases (left panel), or were swapped with males of identical copulation histories and phenotypes in another experimental setting (right panel; Experiment 3). Thus, in the left panel female were given the choice to copulate with one of the two males that they watched during the demonstration phase (this duplicate the situation of Fig. 3 left bars), while in the right panel males had had the same experience during demonstration but were unknown to the observer female. The demonstration phase was the same in both cases: females were allowed to watch Mated males (i.e. that copulated with a virgin female) and Unmated males (i.e. that were rejected by a refractory female) in the two peripheral vials. Females did not prefer the Unmated males over the Mated males when the males were changed while they did so when males were not changed (GLM, male type $\times$ treatment: $\left.\mathrm{Chi}^{2}{ }_{1}=5.59, \mathrm{p}=0.0181\right)$. The horizontal dashed line corresponds to random mate choice. interaction) could affect male behaviour during the test phase. Indeed, males exposed to a recently mated female usually suppress their courtship toward virgin females ${ }^{4-46}$, while a recently mated male does not alter his courting behaviour compared to a virgin male ${ }^{12}$. As such, in our control experiment, males experienced similar interactions with demonstrator females and observer females, but did not have access to visual public information. The results of this control experiment show that public visual cues are necessary to elicit the mating preferences we observed. However, it also suggests that some females may be able to discriminate Mated males against Virgin-control males because Mated males may carry some copulation odours and/or odours of female passively transferred during courting and mating ${ }^{47-48}$. Therefore, in nature, both chemical and visual public information may concur to female avoidance of mated males.

Due to our experiment design, the collection of public information by observer females and the fact that the demonstrator individuals are aware of the presence of an observer (audience effect ${ }^{28}$ ) are confounded and cannot be disentangled. Despite we cannot discard this possibility, we find very unlikely that the audience could drive the results we observed, as it implies that being rejected in front of an observer female should increase male motivation to court this female.

At first glance, our results may appear to contradict previous results by Méry et al. ${ }^{8}$ in which females copied the choice of females they saw copulating. However, Méry et al. ${ }^{8}$ 's experiment differed in two ways. Females successively watched three virgin males of a given phenotype copulating and three virgin males of another phenotype being rejected, and then were given a choice between two new virgin, unfamiliar, males of the two phenotypes. This experiment results in a learnt preference for a category of male (i.e. green or pink male) which imply a cognitive process of phenotype categorization. Rather, in our experiment, observer females were given the choice between the same males that they saw during the demonstration and their discrimination suggests that they were able to discriminate between the two specific males. However, in our experiments 1 and 2, females may have either recognised individual males or just gone for males that looked like the one they observed during the demonstration. The former alternative implies true individual recognition, while the later implies that females only used general characteristics (here the colour of the powder dusting males' bodies) to tell them apart. In the later case, females would be fooled by a pair of males of the same phenotypes, and it was not what we observed. 
NO DELAY

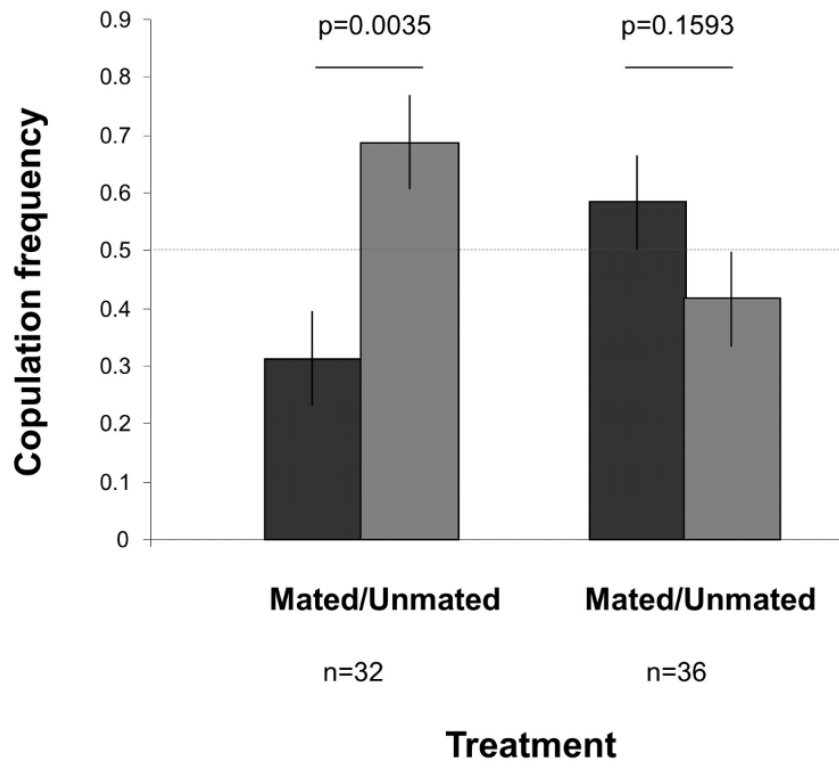

Figure 6 | Female mate choice when there was a 24 hours delay between the demonstration and test phases (Experiment 4). During the demonstration, females were allowed to watch Mated and Unmated males in the two peripheral vials. Mate choice was assessed either immediately (no delay, which triplicate the situation of Fig. 3 left bars) or 24 hours later (24 hours delay). Females avoided Mated over Unmated males immediately after the demonstration but not after a 24 hours delay when they showed an opposite preference (GLM, male type $\times$ treatment: $\left.\mathrm{Chi}^{2}{ }_{1}=10.17, \mathrm{p}=0.0014\right)$. The horizontal dashed line corresponds to random mate choice.

Altogether, our results show that $D$. melanogaster females finetune their use of a piece of information according to the context to make adaptive mating decisions. For instance, they use public information extracted from male copulations to decrease their preference for recently mated males, or to increase their preference for males of the same phenotype when categorisation processes are involved $\left(\right.$ Fig 6 and $\left.^{8}\right)$. Furthermore, our results suggest that female fruit flies have evolved the capacity to visually discriminate between two familiar individuals ${ }^{34}$ and use this capacity to adaptively avoid recently mated males. As our study has been conducted with a laboratory adapted population, and D. melanogaster behaviour is poorly understood in nature, it is unknown whether this behaviour existed in natural populations or recently evolved due to captivity. Therefore, it will be particularly valuable for future research to investigate how fruit flies use public information in the wild.

Our results show that the impressive cognitive abilities (rule learning, concept formation, categorisation and numerical abilities) that have recently been found in insects ${ }^{40-44}$ are used at an unsuspected level of complexity in the context of mate choice, a key component of fitness. They further suggest that flexibility in mate choice mediated by public information is probably a more widespread phenomenon than previously appreciated ${ }^{1,49}$ and support the recently proposed hypothesis that cognitive abilities could have evolved, at least partially, under sexual selection ${ }^{50-51}$.

\section{Methods}

Culture stocks. We used D. melanogaster of wild type Chavroche line obtained from a large outbred laboratory population. Flies were maintained in air-conditioned room at $23^{\circ} \mathrm{C}$ on a $12: 12 \mathrm{~h}$ light:dark cycle and in standard $8 \mathrm{ml}$ vials containing cornmealagar-yeast medium. The population stock was kept at low density by placing 5 mated females (obtained from different vials) for 4 days in a $8 \mathrm{ml}$ vial. Mated females were obtained by placing together about 20 individuals (sex ratio 50/50) each of them obtained from a different parental vial to maximize outbreeding. Emerging offspring were removed daily from the vial in which they hatched. All flies involved in the experiments were 3 days old and used only once. Virgin flies were obtained by separating males and females at emergence, and these flies were kept on food in unisex group of 10-15 individuals. All flies were manipulated by gentle aspiration. We did not use anaesthesia as it may affect memory (e.g. ${ }^{52}$ ).

Female fitness in relation to previous male mating experience. We first investigated whether male fruit flies become semen-limited. Each experimental male was placed in a $1 \mathrm{ml}$ vial containing one virgin female. Vials where no copulation occurred within 30 min were discarded. After copulation, the male was transferred into a new vial containing another virgin female. Males experienced with mating are known to become choosier (e.g. ${ }^{53}$ ), we thus introduced a second female in the vial when copulation did not occur within 30 minutes. This was repeated a third and, a fourth time, and then a fifth time 24 hours later (for a subsample). We recorded the latency and the duration of all four successive copulations. Males who took longer than 1 hour between two copulations were not included in the analyses. Each female was kept in an individual $8 \mathrm{ml}$ vial containing food where she could lay her eggs for 4 days. We counted the number of offspring that subsequently emerged from each vial. A sub-group of 46 females was kept isolated until death to confirm that this number accurately reflects the life-time number of offspring $(n=46$, Spearman rank correlation $\mathrm{r}=0.86, \mathrm{p}<0.0001)$.

Experiment 1: Test for sperm-limitated males avoidance. Experiment 1 was designed to investigate female mate choice when given the opportunity to collect visual public information and to control for chemical cues potentially carried by males. Twelve virgin males were marked using colour powders as in Méry et al. ${ }^{8}$, half in green half in pink. Coloration has no impact on female mate choice ( $n=78$, GLM, $\left.\mathrm{Chi}^{2}{ }_{1}=0.00, \mathrm{p}=1.00\right)$. The six males were put together into a vial containing traces of powder and then transferred into a clean vial containing food where they stayed for at least $45 \mathrm{~min}$ before experiments. This delay allowed them to clean so that only few colour spots dusted their thorax and back at the onset of the experiment. Males were randomly assigned to one of the three following treatments: Mated, Unmated, or Virgin-control.

The experimental set up consisted in three transparent plastic vials providing three compartments separated by two removable transparent microscope cover glass so that the observer female in the central compartment could watch the individuals placed in the two peripheral compartments (Fig. 1). Each $3.3 \mathrm{~cm}$ long and $1 \mathrm{~cm}$ diameter vial was removable and fixed on the base by a clip and contained no food. Six set ups were used in parallel and were visually independent. Vials and cover glasses were cleaned with absolute ethanol after each experiment. This experimental set up was used in Experiments 1, 2, 3 and 4.

Each experiment had a demonstration and a test phase. There were three kinds of demonstration treatments in the peripheral vials. Mated males: a virgin male with a virgin female, which elicited copulation. Unmated males: a virgin male with a refractory female who just copulated; such males courted the female but did not achieve copulation. Virgin males: a virgin male alone. We created all the possible kinds of contrasts between the two peripheral vials: 1) a Mated versus a Unmated male, 2) a Virgin-control versus a Unmated male, or 3) a Virgin-control versus a Mated male. Male types were alternatively placed in the left or right vials and were alternatively pink or green.

After completion of the copulations (i.e. around 40-45 min after the beginning of the demonstration period), we removed the females in the lateral vials, and started the copulation test by removing the two separating cover glasses. The observer female was then able to copulate with one of the two males previously watched, which we recorded.

Experiment 2: Test for visually-acquired information. This experiment was run in parallel with Experiment 1 to test whether visually-acquired public information is sufficient to explain mate choice. In this second experiment, observer females were placed in the experimental set up during the demonstration period and the lateral vial were left empty and the same demonstrations were realized in separated set ups, so that observer females could not watch them. Males were transferred in the lateral vials before the beginning of the test.

Experiment 3: Male-identity recognition. This experiment investigates whether results of Experiment 1 were driven by a preference for a given male phenotype (green versus pink) or by a preference for familiar males. The demonstration protocol was the same as in the With information treatment of Experiment 1 except that we only used Mated and Unmated males. There were two treatments: one that duplicated the Mated/Unmated contrast as in Experiment 1 (Same males); and one in which we swapped males from two parallel set ups at the end of the demonstration (Different males), so that males during the test phase had the same colour and social and mating history but their mating history was unknown to the observer female.

Experiment 4: Semen limitation versus sexually transmitted disease avoidance. This design investigates whether female preferences observed in Experiment 1 were driven by semen-limitation avoidance and/or risk to contract sexually transmitted diseases. The demonstration protocol was the same as for in Experiment 2. However, this time the test phase occurred either immediately after the demonstration as in all previous experiments or only 24 hours later ( 24 hour delay). During the 24 hour 
delay, males and observer females were individually kept in $8 \mathrm{ml}$ vials containing food.

Statistical analyses. We analyzed female mate choice with SAS 9.1.3 (Cary, USA). We used generalized linear models (GLM, proc GENMOD) with a binomial distribution of error terms $(0 / 1=$ unsuccessful/successful, link function: logit). The explanatory variables were the male type (Mated, Unmated or Virgin-control) Represented values are means \pm standard errors. Standard errors of proportions were calculated as the

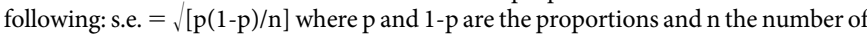
replicates.

1. Chaine, A. S. \& Lyon, B. E. Adaptive plasticity in female mate choice dampens sexual selection on male ornaments in the lark bunting. Science 319, 459-462 (2008).

2. Servedio, M. R., Saether, S. A. \& Saether, G.-P. Reinforcement and learning. Evol. Ecol. 23, 109-123 (2009).

3. Bro-Jørgensen, J. Dynamics of multiple signalling systems: animal communication in a world in flux Trends Ecol. Evol. 25(5), 292-300 (2010).

4. Bailey, N. W. Mate choice plasticity in the field cricket Telegryllus oceanicus: effects of social experience in multiple modalities. Behav. Ecol. Sociobiol. 65, 2269-2278 (2011).

5. Rieucau, G. \& Giraldeau, L.-A. Exploring the costs and benefits of social information use: an appraisal of current experimental evidence. Phil. Trans. $R$. Soc. B 366, 949-957 (2011).

6. Vakirtzis, A. Mate choice copying and nonindependent mate choice: a critical review. Ann. Zool. Fennici 48, 91-107 (2011).

7. Pruett-Jones, S. Independent versus nonindependent mate choice: do females copy each other? Am. Nat. 140, 1000-1009 (1992).

8. Méry, F., Varela, S. A. M., Danchin, É., Blanchet, S., Parejo, D., Coolen, I. \& Wagner, R. H. Public versus personal information for mate copying in an invertebrate. Curr. Biol. 19, 1-5 (2009).

9. Wedell, N., Gage, M. J. G. \& Parker, G. A. Sperm competition, male prudence and sperm-limited females. Trends Ecol. Evol. 17(7), 313-320 (2002).

10. García-González, F. Infertile matings and sperm competition: the effect of "nonsperm representation" on intraspecific variation in sperm precedence patterns. Am. Nat. 164(4), 457-472 (2004).

11. Lefevre, G. Jr. \& Jonsson U.B. Sperm transfer, storage, displacement, and utilization in Drosophila melanogaster. Genetics 47, 1719-1736 (1962)

12. Markow, T. A., Quaid, M. \& Kerr, S. Male mating experience and competitive courtship success in Drosophila melanogaster. Nature 276, 821-822 (1978).

13. Hihara, F. Effects of the male accessory gland secretion on oviposition and remating in females of Drosophila melanogaster. Zool. Mag. 90, 307-316 (1981).

14. Chapman, T. Seminal fluid-mediated fitness traits in Drosophila. Heredity 87, 511-521 (2001).

15. Linklater, J. R., Wertheim, B., Wigby, S. \& Chapman, T. Ejaculate depletion patterns evolve in response to experimental manipulation of sex ratio in Drosophila melanogaster. Evolution 61, 2027-2034 (2007).

16. Sirot, L., Buehner, N., Fiumera, A. \& Wolfner, M. Seminal fluid protein depletion and replenishment in the fruit fly, Drosophila melanogaster: an ELISA-based method for tracking individual ejaculates. Behav. Ecol. Sociobiol. 63, 1505-1513 (2009).

17. Arnquist, G. \& Nilsson, T. The evolution of polyandry: multiple mating and female fitness in insects. Anim. Behav. 60, 145-164 (2000).

18. Jennions, M. D. \& Petrie, M. Why do females mate multiply? A review of the genetic benefits. Biol Rev. 75(1), 21-64 (2000).

19. Rolff, J. \& Siva-Jothy, M. T. Copulation corrupts immunity: a mechanism for a cost of mating in insects. Proc. Natl. Acad. Sci. USA 99, 9916-9918 (2002).

20. Chapman, T., Liddle, L. F., Kalb, J. M., Wolfner, M. F. \& Partridge, L. Cost of mating in Drosophila melanogaster females is mediated by male accessory gland products. Nature 373, 241-244 (1995).

21. Chapman, T., Arnqvist, G., Bangham, J. \& Rowe, L. Sexual Conflict. Trends Ecol. Evol. 18, 41-47 (2003).

22. Arnqvist, G. \& Rowe, L. Sexual Conflict, Princeton University Press, Princeton, NJ. (2005).

23. Fowler, K. \& Partridge, L. A cost of mating in female fruitflies. Nature 338 , 760-761 (1989).

24. Kokko, H. \& Mappes, J. Sexual selection when fertilization is not guaranteed. Evolution 59(9), 1876-1885 (2005).

25. Harris, W. E. \& Moore, P. J. Female mate preference and sexual conflict: females prefer males that have fewer consorts. Am. Nat. 165, S64-S71 (2005).

26. Sato, T. \& Goshima, S. Female choice in response to risk of sperm limitation by the stone crab, Hapalogaster dentata. Anim. Behav. 73, 331-338 (2007).

27. Luck, N. \& Joly, D. Sexual selection and mating advantages in the giant sperm species, Drosophila bifurca. J. Insect Sc. 5, 10 (2005).

28. Danchin, É., Giraldeau, L.-A., Valone, T. J. \& Wagner, R. H. Public information: from nosy neighbors to cultural evolution. Science 305, 487-491 (2004).

29. Wagner, R. H. \& Danchin, É. A taxonomy of biological information. Oikos 119 , 203-209 (2010).

30. Galef, B. G. J. \& White, D. J. Evidence of social effects on mate choice in vertebrates. Behav. Proc. 51, 167-175 (2000).
31. Witte, K. \& Noltemeier, B. The role of information in mate-choice copying in female sailfin mollies (Poecilia latipinna). Behav. Ecol. Sociobiol. 52, 194-202 (2002).

32. Galef, B. G., Lim, T. C. W. \& Gilbert, G. S. Evidence of mate choice copying in Norway rats, Rattus norvegicus. Anim. Behav. 75, 1117-1123 (2008).

33. Kosuda, K. Genetic variability in mating activity of Drosophila melanogaster males. Experientia 39, 100-101 (1983).

34. Ödeen, A. \& Moray, C. M. Drosophila melanogaster virgins are more likely to mate with strangers than familiar flies. Naturwissenschaften 95, 253-256 (2008).

35 . Sheldon, B. C. Sexually transmitted diseased in birds: occurrence and evolutionary significance. Phil. Trans. R. Soc. Lond. B 339, 491-497 (1993).

36. Knell, R. J. \& Webberley, M. Sexually transmitted diseases of insects: distribution, evolution, ecology and host behaviour. Biol. Rev. 79, 557-581 (2004).

37. Miest, T. S. \& Bloch-Qazi, M. C. Sick of mating - Sexual transmission of a pathogenic bacterium in Drosophila melanogaster. Fly 2(4), 215-219 (2008).

38. Gendrin, M., Welchman, D. P., Poidevin, M., Hervé, M. \& Lemaitre, B. Long range activation of systemic immunity through peptidoglycan diffusion in Drosophila. PLoS Pathog. 5(12), e1000694 (2009).

39. Dukas, R. Learning affects mate choice in female fruit flies. Behav. Ecol. 16 800-804 (2005)

40. Menzel, R. \& Giurfa, M. Cognition by a mini brain. Nature 400, 718-719 (1999).

41. Leadbeater, E. \& Chittka, L. Social learning in insects - From miniature brains to consensus building. Curr. Biol. 17, R703-R713 (2007).

42. Chittka, L. \& Niven, J. Are bigger brains better? Curr. Biol. 19, R995-R1008 (2009).

43. Tomchik, S. M. \& Davis, R. L. Out of sight, but not out of mind. Nature 453 , 1192-1194 (2008).

44. Neuser, K., Triphan, T., Mronz, M., Poeck, B. \& Strauss, R. Analysis of a spatial orientation memory in Drosophila. Nature 453, 1244-1247 (2008).

45. Siegel, R. W. \& Hall, J. C. Conditioned responses in courtship behavior of normal and mutant Drosophila. Proc. Natl. Acad. Sci. USA 73, 975-976 (1979).

46. Gailey, D. A., Jackson, F. R. \& Siegel, R. W. Conditioning mutations in Drosophila melanogaster affect an experience-dependent behavioral modification in courting males. Genetics 106, 613-623 (1984).

47. Davis, R. L. The scent of Drosophila sex. Neuron. 54, 14-16 (2007).

48. Everaerts, C., Farine, J.-P., Cobb, M. \& Ferveur, J.-F. Drosophila cuticular hydrocarbons revisited: mating status alters cuticular profiles. PLoS ONE 5(3), e9607 (2010).

49. Bailey, N. W. \& Zuk, M. Field crickets change mating preferences using remembered social information. Biol. Lett. 5, 449-451 (2009).

50. Miller, G. F. Sexual selection for indicators of intelligence, In G. Bock, J. Goode, \& K. Webb (Eds.), The nature of intelligence. Novartis Foundation Symposium 233, John Wiley, pp. 260-275 (2000).

51. Lynn, S. K. Cognition and evolution: learning and the evolution of sex traits. Curr. Biol. 16(11), R421-R423 (2006).

52. Xia, S., Liu, L., Feng, C. \& Guo, A. Memory Consolidation in Drosophila Operant Visual Learning. Learning \& Memory 4, 205-218 (1997)

53. Dukas, R. Male fruit flies learn to avoid interspecific courtship. Behav. Ecol. 15, 695-698 (2004)

\section{Acknowledgements}

We are especially grateful to Fanny Beauché and Pierre Vincent for their help in performing some of the experiments, to Baptiste Logeais, Clémence Fonty and Stéphane Katsoulakis for help in gathering preliminary data, and to Baptiste Logeais for drawing figure 2 . We warmly thank Frédéric Méry, Dominique Joly, Michael Hochberg and Martin Giurfa for valuable comments and scientific discussions on the project, Virginie Népoux and Michèle Huet for technical advices, and two anonymous reviewers for valuable comments on this manuscript. F. Méry kindly provided the coloured powders and the flies. This work was funded by the "Laboratoire d'Excellence (LABEX)" entitled TULIP (ANR -10-LABX-41), and a CNRS post-doc Grant to Adeline Loyau (CR-CDD to JC).

\section{Author contributions}

$\mathrm{AL}$ and PVL collected the data; AL analyzed the data and wrote the first draft of the manuscript; AL, SB, JC and ÉD contributed substantially to design the study, discuss the results and write the manuscript; ÉD initiated and supervised the project.

\section{Additional information}

Competing financial interests: The authors declare no competing financial interests

License: This work is licensed under a Creative Commons

Attribution-NonCommercial-NoDerivative Works 3.0 Unported License. To view a copy of this license, visit http://creativecommons.org/licenses/by-nc-nd/3.0/

How to cite this article: Loyau, A., Blanchet, S., Van Laere, P., Clobert, J. \& Danchin, E. When not to copy: female fruit flies use sophisticated public information to avoid mated males. Sci. Rep. 2, 768; DOI:10.1038/srep00768 (2012). 